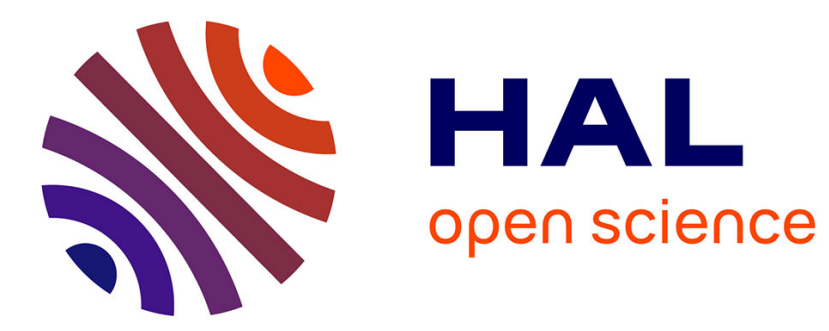

\title{
A mouse sensor and a 2-pixel motion sensor exposed to continuous illuminance changes
}

Fabien Expert, Stéphane Viollet, Franck Ruffier

\section{To cite this version:}

Fabien Expert, Stéphane Viollet, Franck Ruffier. A mouse sensor and a 2-pixel motion sensor exposed to continuous illuminance changes. IEEE Sensors, Oct 2011, Limerick, Ireland. hal-00713577

\section{HAL Id: hal-00713577 \\ https://hal.science/hal-00713577}

Submitted on 2 Jul 2012

HAL is a multi-disciplinary open access archive for the deposit and dissemination of scientific research documents, whether they are published or not. The documents may come from teaching and research institutions in France or abroad, or from public or private research centers.
L'archive ouverte pluridisciplinaire HAL, est destinée au dépôt et à la diffusion de documents scientifiques de niveau recherche, publiés ou non, émanant des établissements d'enseignement et de recherche français ou étrangers, des laboratoires publics ou privés. 


\title{
A mouse sensor and a 2-pixel motion sensor exposed to continuous illuminance changes
}

\author{
Fabien Expert, Stéphane Viollet, Franck Ruffier \\ Institute of Movement Sciences, Biorobotics, CNRS/Aix-Marseille University, France \\ Email:fabien.expert@univmed.fr
}

\begin{abstract}
Considerable attention has been paid during the last decade to navigation systems based on the use of visual optic flow cues, especially for guiding autonomous robots designed to travel under specific lighting conditions. In the present study, the performances of two visual motion sensors used to measure a local 1-D angular speed, namely (i) a bio-inspired 2-pixel motion sensor and (ii) an off-the-shelf mouse sensor, were tested for the first time in a wide range of illuminance levels. The sensors' characteristics were determined here by recording their responses to a purely rotational optic flow generated by rotating the sensors mechanically and comparing their responses with an accurate rate gyro output signal. The refresh rate, a key parameter for future optic flow-based robotic applications, was also defined and tested in these two sensors. The bio-inspired 2-pixel motion sensor was found to be more accurate indoors whereas the mouse sensor was found to be more efficient outdoors.
\end{abstract}

Index Terms-Elementary motion detector (EMD), Optic-flow, Insect Vision, Mouse sensor, Micro-aerial vehicle (MAV), Test bench.

\section{INTRODUCTION}

Several optic flow-based navigation systems have been developed during the past decade for use onboard terrestrial and aerial robots. Many methods of measuring the visual angular speed have been used for robotic purposes, including the "time of travel" scheme [1] which has been implemented using offthe-shelf photodiodes on both a terrestrial robot [2] and several tethered flying robots [3], [4]. During the past few years, some teams have started using off-the-shelf computer mouse sensors as optic flow sensors. Recently, Chan et al. [5] have partly characterized sensors of this kind, which have also been mounted onboard terrestrial [6], [7] and aerial [8], [9] robotic platforms navigating under constant lighting conditions.

However, very few robotic studies have been published so far to our knowledge in which visual motion sensors have been fully characterized, especially under several decades of illuminance. To fill this gap, we recently developed means of testing the reliability of visual motion sensors in terms of their resolution, accuracy, sensitivity, and invariance to contrast in real environments under a large range of illuminance conditions [10], [11]. In this study, our custom-made motion sensor was compared for the first time with an off-the-shelf mouse sensor, as mouse sensors are being increasingly used in robotic applications as optic flow sensors [9]. It was therefore proposed to determine and compare the output signals obtained with:

- a custom-made, bio-inspired 2-pixel motion sensor [11] based on a combination of (i) an off-the-shelf linearly pre-

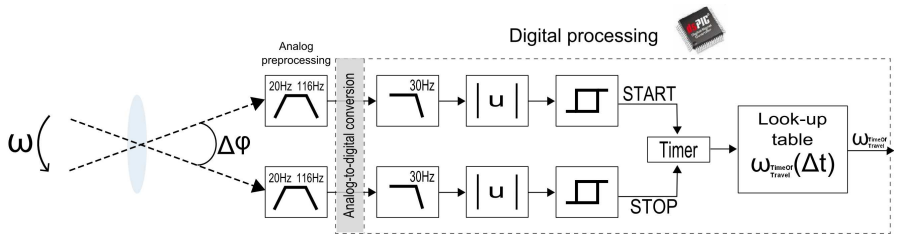

Figure 1. General processing architecture on which the bio-inspired 2pixel motion sensor was based. The output signals emitted by two adjacent pixels were first filtered spatially, via the Gaussian angular sensitivity of the pixels. The two signals were then filtered with an analog band-pass filter with cut-off frequencies of $20 \mathrm{~Hz}$ and $116 \mathrm{~Hz}$ and again with a digital second-order low-pass filter with a cut-off frequency of $30 \mathrm{~Hz}$, before being thresholded to determine the angular speed $\omega_{\text {TimeOfTravel }}$. The "time of travel" scheme previously developed at Franceschini's laboratory [1], [13] was used to measure the angular speed. The overall processing was carried out on a microcontroller (dsPIC 33FJ128GP804) at a sampling frequency of $2 \mathrm{kHz}$ (adapted from [11]).

amplified photodiode array called LSC produced by the company IC-HAUS and (ii) the "time of travel" scheme for processing the angular speed, $\omega_{\text {TimeOfTravel }}$.

- an off-the-shelf mouse sensor ADNS-9500 purchased from Avago, $\omega_{\text {Mouse }}$.

The characteristics of the two motion sensors tested were determined here by recording their responses $\left(\omega_{\text {TimeOfTravel }}\right.$, $\left.\omega_{\text {Mouse }}\right)$ to a purely rotational optic flow generated by rotating the sensors mechanically. In the case of a stationary environment, the rotational optic flow $\omega$, which is by definition independent of the distance from the sensors to the surrounding objects [12], can be directly compared with the rate gyro output signal denoted $\Omega_{\text {gyro }}$.

\section{DESCRIPTION OF THE VISUAL MOTION SENSORS}

\section{A. Bio-inspired 2-pixel motion sensor}

The front end of the bio-inspired motion sensor was based on an off-the-shelf photodiode array called LSC (a component available from iC-Haus) consisting of a row of six pixels with on-chip pre-amplification. Two adjacent pixels separated by an inter-receptor angle $\Delta \varphi$ were combined with a classical fixedgain photocurrent amplifier and used to process the angular speed, based on the "time of travel" scheme [1], [13]. The output signals emitted by these two adjacent pixels were filtered spatially by defocusing the lens (Sparkfun SEN-00637, focal length $2 \mathrm{~mm}$, f-number 2.8) of the sensor in order to give the pixels a similar Gaussian angular sensitivity to that of the fly [14]. These signals were then filtered temporally using a band-pass filter and thresholded to determine the 


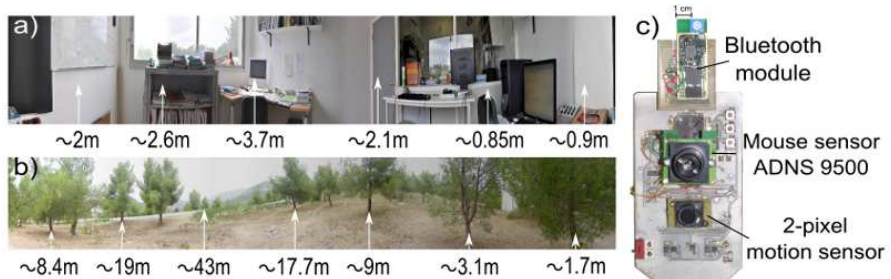

Figure 2. a-b) Picture of the indoor and outdoor scenes where the visual motion sensors were tested. Distances to the surrounding objects are given below. c) Picture of the sensor board consisting of, the bio-inspired 2-pixel motion sensor, the ADNS-9500 mouse sensor, an illuminance sensor based on a single elementary photodiode, a Bluetooth module and a rate gyro.

angular speed $\omega_{\text {meas }}$, defined as the ratio between the constant inter-receptor angle $\Delta \varphi$ and the time $\Delta t$ elapsing between the first and second thresholded signals (see figure 1). More details about this processing system can be found in [11]. We recently designed a stand-alone version of this bio-inspired visual motion sensor (involving a set of 5 neighboring 2-pixel motion sensors) that weighs only $1 \mathrm{~g}$ in all and consumes $75 \mathrm{~mA}$ [15].

\section{B. Mouse sensor (ADNS-9500)}

The ADNS-9500 is a high performance mouse sensor with a frame rate of up to 11750 frames/s, which usually works with a LASER light source. It was equipped here with a Philips CAX100 collimator lens $(\mathrm{f}=10 \mathrm{~mm})$. In a stand-alone version, an overall optic flow sensor device of this kind based on this mouse sensor would probably weigh less than $2 \mathrm{~g}$ and consumes 40mA. The sensor communicated with the microcontroller via SPI bus. The angular speed of the ADNS mouse sensor, which evolved linearly with its output, is given by the following equation

$$
\omega_{\text {Mouse }}=0.025 \times \text { Output }_{A D N S}
$$

where Output $t_{A D N S}$ is the sensor output, and $\omega_{\text {mouse }}$ is the angular speed expressed in $\%$. This coefficient, which was computed to obtain the best correlation between the angular speed measured by the mouse sensor and the reference angular speed $\Omega_{\text {gyro }}$, depends of the focal length of the optical lens $\mathrm{f}$, the frequency of the mouse sensor's readout $f_{R / O}$ and the resolution of the sensor (CPI) expressed in counts per inch. In all the experiments, the data were collected with a readout frequency of $f_{R / O}=25 \mathrm{~Hz}$ and the CPI was left at its default value, 1620.

The quality of the measurements given by the sensor can be assessed using the SQUAL value, which is a register giving the number of valid features detected by the sensor in the current frame. The number of features is given by the equation (2).

$$
\text { Number of features }=S Q U A L \times 4
$$

\section{EXPERIMENTAL SETUP}

The two visual motion sensors were tested indoors and outdoors (see figure 2(a-b)) by comparing their output signals $\left(\omega_{\text {TimeOfTravel }}, \omega_{\text {Mouse }}\right)$ with the angular speed $\left(\Omega_{\text {gyro }}\right)$

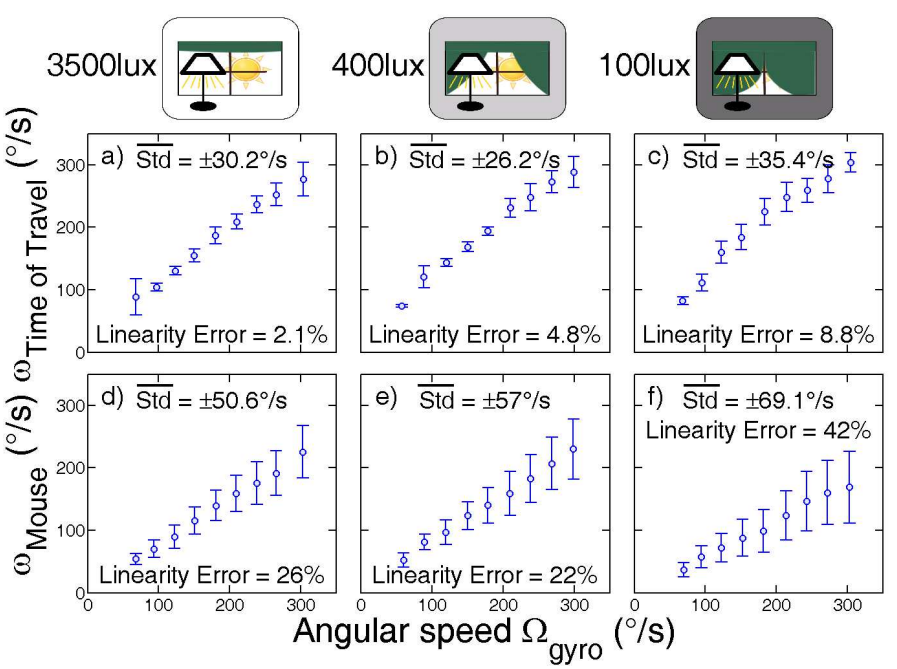

Figure 3. Static characteristics of the visual motion sensors indoors assessed by applying 30-deg/s steps (each lasting $15 \mathrm{~s}$ ) to the rotational speed from 60 to $300 \mathrm{deg} / \mathrm{s}$. (a, b, c) Static characteristics of the bio-inspired 2-pixel motion sensor. (e, f, g) Static characteristics of the ADNS mouse sensor. With both sensors, the linearity error decreased with the illuminance. In all these experiments, the bio-inspired 2-pixel motion sensor proved to be more accurate and linear than the mouse sensor.

measured by a rate gyro (Analog Devices; ADIS 16100), which can be used to measure angular speeds in the 0-300$\mathrm{deg} / \mathrm{s}$ range. The sensor board was connected to a dc motor via a belt, which made it possible to finely adjust the mechanical angular speed of the board in the 60 to $300 \mathrm{deg} / \mathrm{s}$ range.

The static and dynamic responses of the visual motion sensors were measured indoors with several background illuminance values:

- 100 lux corresponding to a dim artificial light.

- 400 lux corresponding to artificial light and a small amount of sunlight from the windows.

- 3,500 lux corresponding to a large amount of sunlight from the windows.

The background illuminance values were measured in lux by a digital luxmeter (Roline; RO-1332), which gives only an estimate of the overall environmental illuminance. The static responses of the sensors were assessed by applying a series of 15-second 30- deg/s steps at a rotational speed ranging from 60 to $300 \mathrm{deg} / \mathrm{s}$. To test the dynamic characteristics of the sensors, a 3x50-s stimulus was applied, during which variably long periods of constantly increasing and decreasing velocities ranging between 60 and $300 \mathrm{deg} / \mathrm{s}$ were imposed.

\section{INDOOR PERFORMANCES OF THE VISUAL MOTION SENSORS}

\section{A. Static angular speed characteristics}

Figure 3 gives the static characteristics of the two sensors tested indoors, as assessed by applying 30- deg/s steps (lasting $15 \mathrm{~s})$ to the rotational speed from 60 to $300 \mathrm{deg} / \mathrm{s}$. The background illuminance ranged between 100 and 3,500 lux. With each visual motion sensor, the mean standard deviation of the data and the linearity error were computed as explained in [11]. 


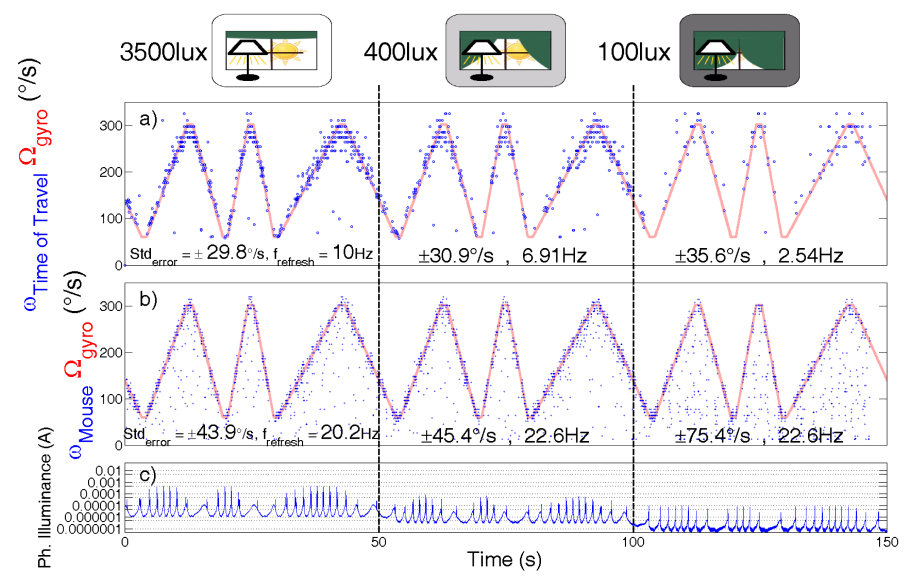

Figure 4. a) Dynamic response of the bio-inspired 2-pixel motion sensor, $\omega_{\text {TimeOfTravel }}$ (blue dots). The sensor can be seen here to have rotated, giving a triangular pattern of variation (red) involving a series of velocity ramps ranging between $60 \%$ and $300 \%$ s with different slopes under three different lighting conditions. Despite the strong illuminance variations, the bioinspired 2-pixel motion sensor followed the rotational angular speed faithfully. The difference between the measured local motion and the angular velocity measured by the rate gyro $\Omega$ gyro was used to compute the standard deviation of the error under each environmental condition. b) Dynamic response of the mouse sensor, $\omega_{\text {Mouse }}$ (blue dots) superimposed on the reference angular speed $\Omega$ gyro measured by the rate gyro. Only measurements differing from zero were plotted. c) Dynamic response of an illuminance sensor based on the use of a single photodiode to measure the effective illuminance of the scene scanned by the visual motion sensors. Strong variations were observed because the light was mainly provided by a single window.

As shown in Figure 3, the output of the bio-inspired 2pixel motion sensor showed a smaller linearity error rate and less dispersion than that of the mouse sensor, which gave a smaller angular speed than the rotational speed imposed on the board. The mouse sensor was designed for use with a LASER; and it was therefore found to be less accurate at low illuminance values than at high ones, even when a large amount of natural light was coming through the windows. The relatively poor linearity of the mouse sensor was only due to the wide dispersion of the data because the mouse sensor's measurement errors were always smaller than the actual value of the angular speed.

\section{B. Dynamic characteristics of the visual motion sensors}

The indoor dynamic responses of both visual motion sensors are shown in figure 4 . The angular speed measured (blue points) is superimposed on the reference angular speed $\Omega$ gyro (red). Despite the continuous changes in the illuminance which can be seen to have occurred in figure 4(c), the response of the bio-inspired 2-pixel motion sensor faithfully obeyed the triangular law imposed on the rotational speed of the board and the dispersion was low. With the mouse sensor, the dispersion increased at low illuminance levels, as can be seen in figure 4(b). The refresh rates of the two sensors were also analyzed, as this is a key parameter in robotic applications. The refresh rate $f_{\text {refresh }}$ of the bio-inspired 2-pixel motion sensor was defined as the number of new measurements divided by the duration of the experiment at each illuminance level, whereas the refresh rate of the mouse sensor was defined as the number of new measurements differing from zero per second.

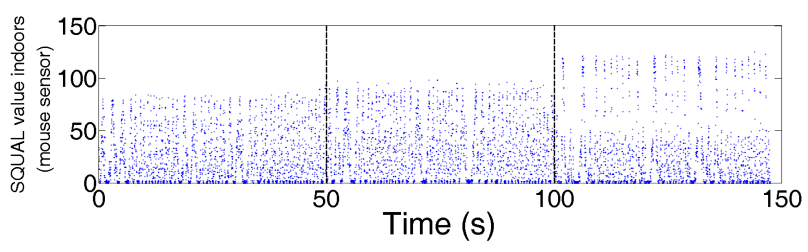

Figure 5. SQUAL value of the mouse sensor (ADNS-9500) during the dynamic experiment indoors. The SQUAL value gives the number of valid features detected by the sensor in the current frame.

As can be seen in figure 4(a), the refresh rate of the bioinspired 2-pixel motion sensor in which the "time of travel" scheme was implemented was found to strongly depend on the illuminance, and decreased from $10 \mathrm{~Hz}$ to $2.54 \mathrm{~Hz}$ between 3,5001 ux and 100lux. The refresh rate of the mouse sensor, on the other hand, was found to be practically independent of the illuminance, and to be greater than that of the bio-inspired 2-pixel motion sensor.

As the SQUAL value gives the number of valid features detected by the sensor in the current frame, it was recorded during the dynamic experiment, as shown in figure 5. As was to be expected, the SQUAL value obtained was often very low $(<10)$, which indicates that the sensor did not detect a sufficiently large number of valid features during the last timestep to achieve a good level of measurement accuracy. The accuracy of the angular speed measurements could certainly be improved by using the sensor output only when the SQUAL value is high enough. Since the large variations observed in the SQUAL values were probably due to the use of a lens with a high F-number, the use of a lens with a smaller F-number should improve the performances of the sensor.

\section{OUTDOOR PERFORMANCES OF THE VISUAL MOTION SENSORS}

When incorporated into a desktop mouse, the mouse sensor is exposed to the strong light emitted by a LASER diode. The mouse sensor's performances could therefore be expected to improve when it was placed in a high illuminance setting. The mouse sensor's dynamic responses were measured in the outdoor environment (see figure 2(b)) on a sunny day under 10000Lux lighting conditions, as shown in figure 6(a). As was to be expected, the dispersion of the data obtained with the mouse sensor was found to be very low $(< \pm 10 \mathrm{~Hz})$ and the refresh rate was equal to the readout frequency. As can be seen in figure 7 , the SQUAL value was always greater than 60 in this case, which means that the sensor computed the angular speed based on a large number of valid features. The outdoor performances of our 2-pixel motion sensor are described in greater detail in [11]. The results obtained here show that the mouse sensor gives better performances under high illuminance conditions than our 2-pixel motion sensor. But the performances of the latter sensor have been improved in recent studies, where the median value of the outputs of five 2-pixel visual motion sensors pointing in different directions were combined and refreshment rates as high as $50 \mathrm{~Hz}$ and a dispersion of only $\pm 10 \%$ were obtained in indoor tests [15]. 


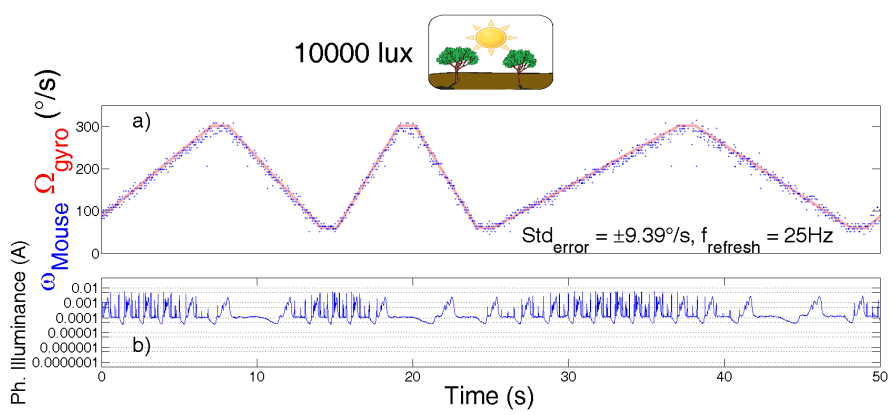

Figure 6. a) Outdoor dynamic response of the mouse sensor, $\omega_{\text {Mouse }}$, under 10000Lux lighting conditions. At high illuminance levels, the data obtained with the mouse sensor showed little dispersion, and the maximum refresh rate possible at this R/O frequency $(25 \mathrm{~Hz})$. b) Dynamic response of the illuminance sensor.

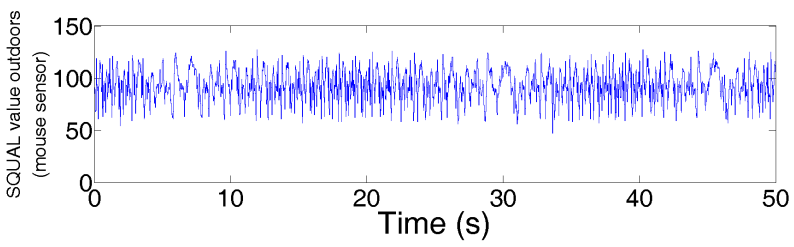

Figure 7. SQUAL value of the mouse sensor (ADNS-9500) during the dynamic experiment outdoors.

\section{CONCLUSION}

In this study, comparisons were made for the first time between the performances of two visual motion sensors used to measure a local 1-D angular speed: a recently developed sensor based on linearly amplified pixels, and a mouse sensor. We showed that our bio-inspired 2-pixel motion sensor in which the "time of travel" scheme was implemented is robust to changes in the illuminance in terms of linearity and dispersion. The angular speed computed indoors by the ADNS 9500 mouse sensor showed important dispersion because too few valid features were detected. However, this mouse sensor gave really accurate, linear and frequently refreshed motion sensing performances under high illuminance conditions, which confirms that this optical sensor is suitable for use on outdoor flying robots. The refresh rates of the two sensors were also analyzed and found to depend slightly on the illuminance in the case of the mouse sensor. In the bio-inspired 2-pixel motion sensor in which the "time of travel" scheme was implemented, the refresh rate was found to strongly depend on the illuminance, but it proved to be suitable for robotic applications where the illuminance is greater than a few hundred Lux, and could therefore be used to pilot the attitude of Micro-Air Vehicles [4]. These findings show that this bioinspired 2-pixel motion sensor can compete satisfactorily with a 900-pixel mouse sensor in terms of its robustness to changes in the illuminance occuring indoors and outdoors, and that it therefore provides a valid alternative to mouse sensors which yield only one scalar measurement per axis of displacement.

\section{ACKNOWLEDGMENT}

We are very grateful to M. Boyron for his help with the electronic design, Y. Luparini and J. Diperi for the mechanical design, F. Roubieu for the panoramic views of the indoor and outdoor environments, J.C. Zufferey, Y. Gasser and A. Briod (EPFL/LIS) for their helpful comments and for providing us with the ADNS9500 equipped with its lens, and Dr. Jessica Blanc for correcting the English manuscript. This work was supported jointly by CNRS Institutes (Life Science; Information Science; Engineering Science and Technology), AixMarseille University II, the French National Research Agency -ANR- (EVA project under ANR-ContInt grant number: ANR08-CORD-007-04) and the European Commission via the CURVACE project. The project CURVACE acknowledges the financial support of the Future and Emerging Technologies (FET) programme within the Seventh Framework Programme for Research of the European Commission, under FET-Open grant number: 237940.

\section{REFERENCES}

[1] C. Blanes, "Appareil visuel elementaire pour la navigation à vue d'un robot mobile autonome." Master's thesis, in Neuroscience, Univ. of Marseille, France, 1986.

[2] N. Franceschini, J. M. Pichon, and C. Blanes, "From insect vision to robot vision," Philosophical Transactions of the Royal Society B: Biological Sciences, vol. 337, pp. 283-294, 1992.

[3] S. Viollet and N. Franceschini, "Super-accurate visual control of an aerial minirobot," in Autonomous Minirobots for Research and Edutainment (AMIRE), vol. Paderborne, Germany, pp. 215-224, Oct 2001.

[4] F. Ruffier and N. Franceschini, "Optic flow regulation: the key to aircraft automatic guidance," Robotics and Autonomous Systems, vol. 50(4), pp. 177-194, 2005

[5] R. Chan, A. Mulla, and K. Stol, "Characterisation of low-cost optical flow sensors," in Proceeding of the Australasian Conference on Robotics and Automation, vol. Brisbane, Australia, pp. 1-8, 2010.

[6] J. D. Jackson, D. W. Callahan, and J. Marstrander, "A rationale for the use of optical mice chips for economic and accurate vehicle tracking," Proc. IEEE International Conference on Automation Science and Engineering CASE 2007, vol. Scottsdale, AZ, USA, pp. 939-944, Sept 2007.

[7] H. Dahmen, A. Millers, and H. A. Mallot, Flying insects and robots. Springer, Berlin, Eds : Floreano, D. and Zufferey,J. C. and Srinivasan, M. V. and Ellington, C., 2009, ch. 9 : Insect inspired odometry by optic flow recorded with optical mouse chips, pp. 115-126.

[8] S. Griffiths, J. Saunders, A. Curtis, B. Barber, T. McLain, and R. Beard, "Maximizing miniature aerial vehicles," IEEE Robotics and Automation Magazine, vol. 13, no. 3, pp. 34-43, Sept. 2006.

[9] A. Beyeler, J.-C. Zufferey, and D. Floreano, "Vision-based control of near-obstacle flight," Autonomous robots, vol. 27, no. 3, pp. 201-219, 2009.

[10] S. Viollet, F. Ruffier, T. Ray, M. Menouni, F. Aubépart, L. Kerhuel, and N. Franceschini, "Characteristics of three miniature bio-inspired optic flow sensors in natural environments," in IEEE International Conference on Sensor Technologies and Applications (SENSORCOMM), pp. 51-55, Venice, Italy, 2010.

[11] F. Expert, S. Viollet, and F. Ruffier, "Outdoor field performances of insect-based visual motion sensors," Journal of Field Robotics, vol. 28 (4), pp. 529-541, 2011.

[12] J. Koenderink and A. Doorn, "Facts on optic flow," Biological Cybernetics, vol. 56, pp. 247-254, 1987.

[13] N. Franceschini, F. Ruffier, J. Serres, and S. Viollet, Aerial vehicles. Vienna : In-Tech, Eds : T. M. Lam, 2009, ch. 35 : Optic flow based visual guidance: from flying insects to miniature aerial vehicles, pp. 747-770.

[14] K. Götz, "Optomotorische Untersuchung des visuellen systems einiger Augenmutanten der Fruchtfliege Drosophila," Biological Cybernetics, vol. 2, pp. 77-92, 1964.

[15] F. Roubieu, F. Expert, M. Boyron, B. Fuschlock, S. Viollet, and F. Ruffier, "A novel 1-gram insect based device measuring visual motion along 5 optical directions," in IEEE Sensors Conference, Limerick, Ireland, 2011 (in press). 